\title{
Timecourse of coactivation in bimodal divided attention
}

\author{
JEFF MILLER \\ University of California, San Diego, California
}

\begin{abstract}
Reaction time distributions were obtained from practiced subjects in a go/no-go detection task with attention divided across the visual and auditory modalities. Redundant signals were sometimes presented asynchronously on the two modalities, with the time between signals varying from 0 to $167 \mathrm{msec}$. An extension of the inequality derived by Miller (1982) was used to test between separate-decisions models, in which the response is initiated solely by whichever signal is detected first, and coactivation models, in which both signals contribute to the activation of a single response. As in previous studies with bimodal detection tasks, the results contradicted separate-decisions models and favored coactivation models. The largest violations of separatedecisions models were observed when the visual signal was presented $67-100 \mathrm{msec}$ before the auditory signal. A new inequality was also derived to discriminate between two classes of coactivation models that differ about whether responses are generated by processes combining activation across time as well as across signals. Violations of this inequality ruled out exponential coactivation models, in which activation processes are sensitive only to the instantaneous properties of the signal(s). Instead, the results require an accumulation model of coactivation, in which both signals provide input to a process that accumulates activation over a considerable period of time, even if signal conditions change during that time.
\end{abstract}

In divided-attention tasks, people are often asked to monitor two different information channels and make a speeded response as soon as a signal is presented on either channel. To understand the division of attention, it is particularly important to determine what happens when subjects must process two signals presented simultaneously on different channels. Intuitively, it seems that the consequences of dividing attention should be most pronounced when both channels require action at the same time, so the characteristics of the underlying division should be particularly apparent under such circumstances.

Empirically, it is almost always the case that a response is made faster when it is indicated by two simultaneously presented signals, one on each channel, than when a single signal is presented on either channel alone (e.g., Raab, 1962). This paper is concerned with the explanation of this advantage, often called the redundant signals effect (RSE), in bimodal detection tasks with visual and auditory signals. The RSE has consistently been obtained in such tasks (e.g., Miller, 1982), but explanations of the effect vary widely.

The simplest explanation of the RSE is embodied in a class of models commonly called separate-decisions or

This research was supported by Grant PHS MH40733 from the National Institute of Mental Health. I would like to thank G. R. Grice, Rolf Ulrich, Patricia Haden, and two anonymous reviewers for helpful comments on earlier versions of the paper, and the two volunteer subjects for their assistance in collecting the data. A preliminary report of this research was presented at a symposium entitled "Action, Attention and Automaticity," held at the Center for Interdisciplinary Research, Bielefeld, Germany, in December 1984. Requests for reprints should be addressed to the author at Department of Psychology, C-009, UCSD, La Jolla, CA 92093. race models (Raab, 1962). In these models, separate processes respond to signals on different channels. When one signal is presented, the response is activated solely by the process that monitors that signal's channel. The process associated with each channel is stochastic, so the response time (RT) to a given signal varies randomly from trial to trial. When both signals are presented simultaneously, each process proceeds as if only its own signal had been presented. If the two processes compete for resources (e.g., attention), their finishing times may be negatively correlated; if they are influenced in the same way by an internal state (e.g., alertness), their finishing times may be positively correlated; or, of course, the finishing times may be independent. In any case, the response is caused by the first of the two separate processes to finish (hence the name "race models"). Assuming that there is some overlap in the distributions of the finishing times for the two response-activation processes so that each wins the race on some of the trials with redundant signals, responses to redundant signals will be faster, on average, than responses to either of the signals presented alone. In this model, the RSE reflects facilitation of a purely statistical variety (Raab, 1962), since the process that activates any given response is influenced by the presence of only one of the two signals.

Models that embody the assumption of separate decisions are plausible candidates to explain the RSE, because they have had considerable success in predicting detection accuracy in bimodal divided attention tasks (e.g., Corcoran \& Weening, 1969; Mulligan \& Shaw, 1980; Shaw, 1982). A variety of evidence contradicts these models, however, by showing interactions among stimuli on differ- 
ent sensory channels (e.g., Bernstein, 1970; Colavita, 1974; Gielen, Schmidt, \& Van den Heuvel, 1983; Long, 1976; Nickerson, 1973). This paper focuses on one particular source of evidence against these models, obtained by examining RT distributions within bimodal dividedattention tasks.

Miller (1982) noted that all race models must make an explicit prediction regarding the distributions of RTs that should be observed in response to redundant signals. Let $\mathrm{RT}_{V}, \mathrm{RT}_{A}$, and $\mathrm{RT}_{R}$ be random variables corresponding to the RTs observed on trials with visual signals, auditory signals, and redundant signals, respectively. $\mathrm{RT}_{V}$ and $\mathrm{RT}_{A}$ might have any bivariate probability distribution, but, according to race models, $\mathrm{RT}_{R}$ is constrained by the relation:

$$
\mathrm{RT}_{R}=\min \left(\mathrm{RT}_{\mathrm{V}}, \mathrm{RT}_{A}\right) .
$$

This relation follows directly from the assumption that the response on a redundant-signals trial is determined by whichever of the separate processes finishes first. Given this constraint, it is easy to prove (see Miller, 1982) that the following inequality must be satisfied by $F_{V}(t)$, $F_{A}(t)$, and $F_{R}(t)$, the cumulative probability density functions (CDFs) of RTs in the three conditions:

$$
F_{R}(t) \leq F_{V}(t)+F_{A}(\mathrm{t}) \text { for all } t \text {. }
$$

That is, for any time, $t$, the proportion of redundant-signal responses faster than $t$ should be less than or equal to the sum of the proportion of visual-signal responses faster than $t$ and the proportion of auditory-signal responses faster than $t$.

Miller (1982) tested and found violations of Inequality 1 in a bimodal detection task with easily detectable visual and/or auditory stimuli. Subjects were required to press a response key as quickly as possible on trials with either the visual signal or the auditory signal, or with both, and to withhold the response on trials without any signal. The observed RTs on trials with visual signals, auditory signals, and redundant signals were used to estimate the respective CDFs $F_{V}(t), F_{A}(t)$, and $F_{R}(t)$, and the estimated values significantly violated Inequality 1 . In particular, the inequality was violated for relatively small values of $t$, suggesting that there were more fast responses to redundant signals than could be explained in terms of a race between separate responses to visual and auditory signals. Thus, race models cannot account for the RSE.

The observed violations of Inequality 1 support models in which the RSE reflects more than just statistical facilitation of average RT. In these models, simultaneous signals on different channels activate the response jointly rather than separately, so the system must combine response activations contributed by processes that monitor different channels. Such models have been referred to as coactivation models (e.g., Grice, Canham, \& Boroughs, 1984; Miller, 1982).

The experiment reported in this paper was designed to study the timecourse of response coactivation in bimodal detection tasks. Redundant visual and auditory signals were presented with signal onset asynchronies (SOAs) up to $167 \mathrm{msec}$, and the resulting data were used to address two major issues about the timecourse of response coactivation.

One issue was whether RTs to asynchronous redundant signals would reveal violations of race models analogous to those observed with simultaneous redundant signals, and, if they did, how the size of the violations would depend on SOA. ${ }^{1}$ To evaluate race models with asynchronous signals, Inequality 1 must be generalized slightly. According to race models, a response to redundant signals is caused by the first to finish of the two separate processes responding to each signal. Obviously, the two processes will not start at the same time if signal presentation is asynchronous, and finishing times must be adjusted to take that fact into account. If RT is measured from the onset of the first signal, the SOA between signals must be added to the latency of responses to the second signal. Let $\mathrm{SOA}_{V}$ and $\mathrm{SOA}_{A}$ denote the SOAs from the onset of the first signal to the onset of the visual and auditory signals, respectively. This notation is very convenient when measuring RT from the onset of the first signal, although it is admittedly peculiar in that one of the two SOAs must equal zero on any trial (i.e., the SOA for the first signal). Given this notation, the race model asserts that:

$$
\mathrm{RT}_{R, \mathrm{SOA}}=\min \left(\mathrm{RT}_{V}+\mathrm{SOA}_{V}, \mathrm{RT}_{A}+\mathrm{SOA}_{A}\right),
$$

where $\mathrm{RT}_{R, \text { soA }}$ is the obtained $\mathrm{RT}$ on a redundant signals trial with the indicated $\mathrm{SOA}$, and $\mathrm{RT}_{V}$ and $\mathrm{RT}_{A}$ are still the finishing times for the separate processes responding to visual and auditory signals. The addition of the SOA is the appropriate correction for asynchronous signals; if the start of one racer is delayed by an amount SOA, that racer's completion time will increase by the same amount. This constraint imposed by race models is exactly analogous to the one with simultaneous redundant signals, and an inequality analogous to Inequality 1 follows immediately:

$F_{R, \mathrm{SOA}}(t) \leq F_{V}\left(t-\mathrm{SOA}_{V}\right)+F_{A}\left(t-\mathrm{SOA}_{A}\right)$ for all $t$.

Thus, one purpose of the present research was to see whether Inequality 2 was violated-and race models further contradicted-with asynchronous redundant signals.

The second issue addressed by the present research involved a comparison between two classes of coactivation models using a new inequality related to Inequalities 1 and 2. Although those inequalities can be used to see whether response processes combine activation across signals, the second main objective of this study was to investigate the question of whether response processes could combine activation across time. Two classes of coactivation models were considered-referred to here as "accumulation" and "exponential" models - both of them generalizations of standard RT models developed for tasks with single stimuli and no attentional manipulations. Both classes of models are consistent with violations of Inequality 1 , but only the former allows for a combination 
of activation across time. In brief, accumulation models assume that coactivation occurs in a process that builds up response activation gradually over time, whereas exponential models assume that coactivation occurs in a process that changes states in an instantaneous, all-or-none fashion to generate a response.

Accumulation models are based on a mechanism that sums small activations gradually over time, stopping when a criterion is reached. This class of coactivation models is a natural extension of RT models-often based on statistical decision theory (e.g., Audley, 1973)-in which a decision process accumulates evidence that a signal has been presented (i.e., "response activation"), emitting the response when the amount of activation reaches a certain criterion level (e.g., Green \& Smith, 1982; Link, 1975). To account for divided-attention tasks, accumulation models can be generalized to allow activation from more than one channel to contribute to the process accumulating activation. Such a model can easily explain the RSE, because summing of activations produced by two signals on different channels would allow the stopping criterion to be satisfied before either signal had produced enough activation to satisfy the criterion by itself. In essence, the inequality predicted by race models is violated because of a change in the rule for stopping the race. In an accumulation model the race stops when the total distance covered by the two runners is equal to the criterion distance, whereas in a race model it stops as soon as one of the runners covers the criterion distance. ${ }^{2}$

In coactivation models of the exponential class, redundant signals combine in their influence on the instantaneous state of the process generating the response, but there is no accumulation of activation or evidence over time. This class of coactivation models is an extension of RT models with exponentially distributed decision times (e.g., Ashby, 1982; Ashby \& Townsend, 1980; Christie \& Luce, 1956; Green \& Smith, 1982; Hockley, 1984; Hohle, 1965; Luce \& Green, 1970; Ratcliff, 1978; Ratcliff \& Murdock, 1976; Townsend \& Ashby, 1983), because in these models the decision to respond is made by a mechanism that has a constant instantaneous probability (i.e., rate) of terminating at each moment after it begins (see McGill, 1963). It is usually assumed that total RT is the sum of an exponentially distributed decisionmaking time and a normally distributed residual time that reflects sensory and motor processes.

To extend exponential models to divided attention tasks, one can allow the rate of the decision process to depend on the signal configuration, with one rate for a visual signal, a second rate for an auditory signal, and a third rate for redundant signals. More specific exponential models could attempt to specify the rate for redundant signals as a function of the rates for the two individual signals. In general, though, exponential models are consistent with the RSE as long as the rate for redundant signals is higher than the rate for either of the individual signals. Furthermore, they are consistent with violations of Inequality 1 if the rate for redundant signals is sufficiently greater than the sum of the rates for the two individual signals.

To sharpen the contrast between accumulation and exponential models and hint at a way of deciding between them, note that an exponential process can be considered history-free, because the probability of finishing in the next instant of time does not depend on how long the process has been active (i.e., its history). Accumulation models, on the other hand, are history-dependent in the sense that the probability of finishing in the next instant increases with the time since the process began.

The SOA manipulation can be used to discriminate between accumulation and exponential models by revealing whether responses are jointly activated by both the single signal present initially and the redundant signals present after the end of the SOA. Such an effect is required by history-dependent accumulation models, but is inconsistent with history-free exponential models. According to accumulation models, the first signal must start contributing increments of activation that move the accumulator some of the way toward the criterion. Once the second signal is presented, both signals contribute additional increments toward the criterion. This means that the criterion is satisfied partly by activation from the first signal alone and partly by activation from the two signals acting together. In the present analysis, the increments provided by the first signal can be regarded as the history of the accumulation process, and the amount of activation that must be accumulated from the two signals acting together (i.e., starting at the end of the SOA) is influenced by this history.

In contrast, exponential models require the response to be produced entirely by one of the instantaneous states of the system. This could be the state produced by the first signal, present until the end of the SOA, or the state produced by the redundant signals, present after the end of the SOA. Since there is no accumulation across time in history-free exponential models, however, the response cannot be influenced both by the presence of the first signal and by the presence of the redundant signals.

In essence, then, the distinction between accumulation and exponential models is that the former allow the response to be influenced by two different signal configurations that occur sequentially on a single trial, whereas the latter allow the response to be influenced by only one of them. ${ }^{3}$ This distinction in the time domain is analogous to the distinction in the signal domain between race models and coactivation models: coactivation models allow combination of activation across signals, whereas race models do not; analogously, accumulation models allow combination of activation across time, whereas exponential models do not. This analogy suggests that exponential models must make a prediction similar to Inequalities 1 and 2.

Consider a trial on which a visual signal is presented first and an auditory signal is presented after some nonzero $\mathrm{SOA}_{A}$. According to exponential models, a response 
observed on this trial will have been activated either by the visual signal present before the end of the $\mathrm{SOA}_{A}$ or by the redundant signals present after the end of the $\mathrm{SOA}_{A}$, but not by both. ${ }^{4}$ This situation is almost analogous to a race between two response processes, with the process responding to the visual signal getting a head start equal to the $\mathrm{SOA}_{A}$. If the race analogy held exactly, then exponential models would assert that

$$
\mathrm{RT}_{R, \mathrm{SOA}}=\min \left(\mathrm{RT}_{V}, \mathrm{RT}_{R, 0}+\mathrm{SOA}_{A}\right),
$$

where $\mathrm{RT}_{R, 0}$ is the $\mathrm{RT}$ to simultaneous redundant signals.

The race analogy does not hold exactly, however, since the racer for the visual signal is replaced by the racer for the redundant signal if the former has not finished when the redundant signal starts. Forcing the visual racer to drop out when the redundant racer starts can only slow down the winning time, relative to what would be observed if the visual racer continued in the race. Thus, exponential models actually assert that

$$
\mathrm{RT}_{R, \mathrm{SOA}} \geq \min \left(\mathrm{RT}_{V}, \mathrm{RT}_{R, 0}+\mathrm{SOA}_{A}\right) .
$$

This constraint leads immediately to Inequality 3 , by direct analogy to Inequalities 1 and 2:

$$
F_{R, \mathrm{SOA}}(t) \leq F_{V}(t)+F_{R, 0}\left(t-\mathrm{SOA}_{A}\right) \text { for all } t \text {. }
$$

The interpretation of Inequality 3 is that the response on a trial with a visual signal followed after $\mathrm{SOA}_{A}$ msec by an auditory one should take long enough for it to be attributable either to the visual signal state present at the start of the trial or to the redundant signal state appearing $\mathrm{SOA}_{\mathrm{A}}$ msec later. An analogous inequality can be constructed for trials on which the auditory signal is presented first.

To summarize, the second purpose of this experiment was to obtain data that could be used to test Inequality 3 . This inequality can be used to test exponential coactivation models, all of which assert that it should be satisfied. Because it is an inequality, the test is rather conservative; however, if any data are found to be inconsistent with the inequality, the entire class of models can be rejected.

\section{METHOD}

The apparatus and signals were the same as those used by Miller (1982, Experiments 1 and 2): visual signals were responseterminated plus signs appearing in the center of a computer display screen, and auditory signals were $780-\mathrm{Hz}$ tones of $150-\mathrm{msec}$ duration. Responses were made by pressing the / (slash) key on the computer keyboard with the right index finger. Two naive volunteer subjects, a 35-year-old male (B.D.) and a 21-year-old female (K.Y.), were tested over a period of about 1 month. Both were righthanded. Each was tested in 40-min sessions during which two blocks were completed, with one session per day and four to six sessions per week. Subject B.D. eventually completed 42 blocks, and Subject K.Y. completed 43 . Only the last $\mathbf{4 0}$ blocks completed by each subject were included in the analysis.

Each bimodal detection trial began when the subject pressed a key to indicate readiness. Then a fixation point was presented for $250 \mathrm{msec}$ and there was a random foreperiod lasting from 250 to
$2,250 \mathrm{msec}$ (uniformly distributed). At that point, the first signal was presented, and after an appropriate SOA, the second signal (if any) was presented unless the subject had already responded. On catch trials, there was a 2-sec blank interval at the end of the random foreperiod, during which false alarms were recorded. No feedback was given after responses. After each response there was an enforced delay of $500 \mathrm{msec}$, after which the subject was given the opportunity to initiate the next trial by pressing a key. Any trial on which an error (false alarm or miss) occurred was renun later in the block.

Each block started with 4 warm-up trials, followed by 170 test trials. There were 40 catch trials, on which no signal was presented and no response was to be made, and 130 signal trials. The latter 130 trials were equally divided among 13 conditions: (1) visual signal alone, (2) auditory signal alone, (3) both signals simultaneously, (4-8) visual signal presented first, then auditory signal presented $33,67,100,133$, or $167 \mathrm{msec}$ later $\left(\mathrm{SOA}_{A}\right)$, and $(9-13)$ auditory signal presented first, then visual signal presented $33,67,100,133$, or $167 \mathrm{msec}$ later $\left(\mathrm{SOA}_{v}\right)$. The subjects were instructed to respond as quickly as possible if either the visual or auditory signal was presented, or both, but to withhold the response if no signal was presented.

\section{RESULTS AND DISCUSSION}

Before proceeding with the analysis of models using Inequalities 2 and 3 , it is worthwhile to examine several features of the basic results. Table 1 shows descriptive statistics for RT as a function of SOA, with SOAs ordered symmetrically around the condition of simultaneous signals. One of the most clear-cut results was that responses to single auditory signals were much faster than those to single visual signals [B.D., $t(798)=21, p<.01 ; \mathrm{K}$.Y., $t(798)=16.2, p<.01]$. Faster responding to auditory signals also appears to have influenced the results obtained with redundant signals in three respects. First, there was very little effect of a redundant visual signal presented after the auditory signal, since responses to an auditory signal presented alone were as fast as responses to an auditory signal followed by a visual signal in all but one comparison [B.D., $\mathrm{SOA}_{V}=33, t(798)=3.27, p<.01$ ]. This probably results from faster responding to auditory than to visual signals, because delaying the more slowly processed visual signal would make it arrive too late to have any impact on the response to the more quickly processed auditory signal.

Second, auditory signals influenced responses even when they were presented long after visual signals. Mean RT increased systematically with $\mathrm{SOA}_{A}$, the time from the visual to the auditory signal. For Subject B.D., responses were faster when the auditory signal was presented $167 \mathrm{msec}$ after the visual signal than when the visual signal was presented alone, a particularly surprising result because of the speed of responses in a simple RT task. This effect is probably also related to the singlesignal advantage for auditory signals, since a more quickly processed auditory signal could often overtake a more slowly processed visual one, even when the latter had a head start.

Third, a relatively small RSE was obtained when visual and auditory signals were presented simultaneously. This 
Table 1

Descriptive Statistics as a Function of SOA and Subject

\begin{tabular}{|c|c|c|c|c|c|c|c|c|c|c|c|c|c|}
\hline & \multicolumn{13}{|c|}{ SOA Condition } \\
\hline & \multicolumn{6}{|c|}{ Auditory First: } & \multirow[b]{3}{*}{0} & \multicolumn{6}{|c|}{ Visual First: } \\
\hline & \multirow[b]{2}{*}{ Alone } & \multicolumn{5}{|c|}{$\mathrm{SOA}_{4}$} & & \multicolumn{5}{|c|}{ SOA } & \multirow[b]{2}{*}{ Alone } \\
\hline & & 167 & 133 & 100 & 67 & 33 & & 33 & 67 & 100 & 133 & 167 & \\
\hline \multicolumn{14}{|c|}{ Subject B.D. } \\
\hline Mean RT & 231 & 234 & 230 & 227 & 228 & 221 & 217 & 238 & 263 & 277 & 298 & 316 & 348 \\
\hline$S E$ & 2.8 & 2.9 & 2.0 & 2.0 & 1.6 & 1.4 & 1.4 & 1.4 & 1.3 & 1.5 & 1.6 & 1.7 & 4.6 \\
\hline Min RT & 164 & 180 & 162 & 165 & 155 & 166 & 158 & 189 & 213 & 215 & 93 & 235 & 60 \\
\hline Median RT & 219 & 223 & 222 & 221 & 223 & 219 & 215 & 233 & 259 & 274 & 296 & 316 & 326 \\
\hline Max RT & 720 & 966 & 403 & 624 & 347 & 437 & 469 & 546 & 431 & 648 & 526 & 532 & 767 \\
\hline RSE & & -3 & 1 & 4 & 3 & $10^{*}$ & $14 *$ & $26^{*}$ & $35^{*}$ & $54 *$ & $50 *$ & $32 *$ & \\
\hline \multicolumn{3}{|c|}{$\%$ Misses $=0.0$} & \multicolumn{4}{|c|}{$\%$ False Alarms $=0.1$} & \multicolumn{7}{|c|}{$N=400$ observations per SOA } \\
\hline \multicolumn{14}{|c|}{ Subject K.Y. } \\
\hline Mean RT & 211 & 216 & 217 & 214 & 218 & 215 & 208 & 237 & 249 & 256 & 273 & 278 & 282 \\
\hline$S E$ & 3.0 & 3.7 & 3.8 & 3.9 & 3.8 & 3.3 & 3.2 & 3.1 & 2.9 & 2.3 & 2.7 & 3.1 & 3.1 \\
\hline Min RT & 138 & 130 & 131 & 144 & 58 & 140 & 88 & 176 & 79 & 166 & 113 & 192 & 196 \\
\hline Median RT & 193 & 196 & 194 & 192 & 192 & 198 & 190 & 216 & 235 & 244 & 264 & 268 & 266 \\
\hline $\operatorname{Max} R T$ & 499 & 741 & 544 & 1060 & 489 & 489 & 502 & 557 & 560 & 542 & 585 & 612 & 664 \\
\hline RSE & & -5 & -6 & -3 & -7 & -4 & 3 & 7 & $29 *$ & $26 *$ & 9* & 4 & \\
\hline \multicolumn{3}{|c|}{$\%$ Misses $=0.2$} & \multicolumn{4}{|c|}{$\%$ False Alarms $=1.3$} & \multicolumn{7}{|c|}{$N=400$ observations per SOA } \\
\hline
\end{tabular}

Note-SE $=$ standard error of the mean. $\quad{ }^{*} \mathrm{p}<.05$.

is probably also due to the faster processing of auditory signals, because a quickly processed auditory signal would usually have time to evoke the response before the processing of a simultaneous visual signal had made much contribution.

Table 1 also shows, for each subject, the RSE computed for each of the redundant-signals conditions, with asterisks marking those that were significantly different from zero by a two-sample $t$ test. The RSE with simultaneous signals is calculated as

$$
\mathrm{RSE}=\min \left(\overline{\mathrm{RT}}_{V}, \overline{\mathrm{RT}}_{A}\right)-\overline{\mathrm{RT}}_{R} .
$$

With asynchronous signals, the average RTs must be corrected for the late onset of the second signal, as discussed in the introduction:

$\mathrm{RSE}_{\mathrm{SOA}}=\min \left(\overline{\mathrm{RT}}_{\mathrm{V}}+\mathrm{SOA}_{V}, \overline{\mathrm{RT}}_{A}+\mathrm{SOA}_{A}\right)-\overline{\mathrm{RT}}_{R}$, SOA.

It is apparent that the RSE was replicated in this experiment. In fact, the largest benefits for redundant signals occurred when the auditory signal was presented 67 - $133 \mathrm{msec}$ after the visual signal. This pattern is also qualitatively consistent with the fact that the auditory signal was processed faster; the visual signal must be given a head start to enable it to have much influence on the response. A somewhat surprising difference between subjects was that B.D. showed much larger RSEs than did K.Y. The source of this individual difference was not clear from subjective reports, and Subject K.Y. appears to be somewhat atypical in view of the consistency with which RSEs are obtained.

\section{Test of Race Models Using Inequality 2}

Inequality 2 was used to see whether the predictions of race models were violated when redundant signals were asynchronous. To use the inequality, it is necessary to estimate $F_{V}(t), F_{A}(t)$, and the 11 different $F_{R \text {,soA }}(t)$ functions. These CDFs were estimated separately for each subject by pooling together all of the RTs from the appropriate condition into the necessary cumulative distribution.

After the various CDFs were thus estimated, Inequality 2 was used to check for violations of race models in these data. For each of the 11 redundant signal conditions, the observed $F_{R, \text { SOA }}(t)$ was compared against the sum of $F_{V}\left(t-\mathrm{SOA}_{V}\right)$ and $F_{A}\left(t-\mathrm{SOA}_{A}\right)$. For example, Figure 1 shows these CDFs for Subject B.D. in the condition with an $\mathrm{SOA}_{A}$ of $100 \mathrm{msec}$. As is apparent in this figure, Inequality 2 was seriously violated at this SOA, since the observed redundant-signals CDF is considerably above the sum of the single-signal CDFs for almost all of the values of RT observed in response to redundant signals. This figure was chosen to illustrate the nature of violations of

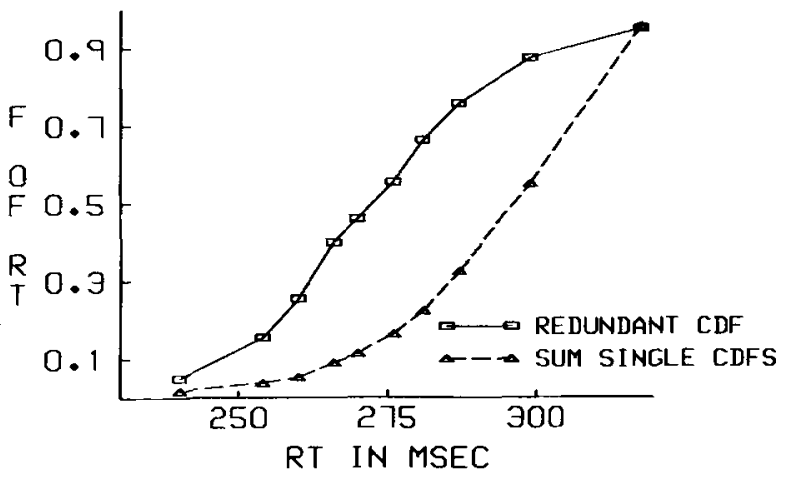

Figure 1. Subject B.D. Observed CDF of RTs to redundant signals with visual signal presented first and $S O A_{A}=100$, compared against the sum of the CDFs of RTs to the two single signals [i.e., $\left.F_{v}(t)+F_{A}(t-100)\right]$. 
this inequality, and it is one of the ones showing the largest violations. It is interesting to note that many more responses were observed in the fastest latency range (e.g., $\mathrm{RT}<270 \mathrm{msec}$ ) than can be predicted by race models. This suggests that the fastest responses to redundant signals can sometimes be faster than the fastest responses to either of the single signals, contrary to race models. Unfortunately, it is difficult to test for this effect directly using minimum RTs, because anticipations (i.e., the processes that produce false alarms on trials without a signal) almost certainly produce a few spuriously fast RTs in all conditions.

In order to present the violations of Inequality 2 for both subjects and all SOAs without including the 22 different figures corresponding to Figure 1, it is convenient to use a single numerical measure of the size of the violation at each SOA. In Figure 1, the violation of the inequality is reflected in the fact that there is a large area below the curve defined by $F_{R, 100}(t)$ but above the curve defined by the sum of $F_{V}(t)$ and $F_{A}(t-100)$. This area is readily quantified numerically, and it seems the most direct summary measure of the extent to which Inequality 2 is violated at each SOA. Standard measures for comparing distributions (e.g., Kolmogorov-Smirnoff) would not be appropriate in this case, since the prediction being examined is an inequality rather than an equality. For the present analyses, the area of violation was measured as a proportion of the total area under each $F_{R, \operatorname{soA}}(t)$. The $V$ (Observed) values in Table 2 are these area measures [in units of $1 / 1,000$ the total area under $F_{R, \mathrm{SOA}}(t)$ ], and they are intended to reflect the extent to which Inequality 2 was violated for each of the different subjects and conditions.

The $V($ Observed) values in Table 2 contradict race models by showing substantial violations of Inequality 2 . As much as half of the total area under the observed CDFs for redundant signals lies outside the range compatible with race models, and violations of at least $10 \%$ of the total area are not uncommon. It is not surprising that the violations of Inequality 2 are largest for conditions in which the visual signal was presented first. This finding is consistent with the observation that auditory signals were processed much faster, since an initial auditory signal could produce a response before the later visual signal had time to have any influence at all. Clearly, the coactivation reported by Miller (1982) seriously underestimated the true potential for coactivation in this task, since these same signals were presented simultaneously in the earlier experiment.

The obtained pattern of $V$ (Observed) values is also strong evidence against the idea that race models can explain the RSE, because race models were seriously violated at precisely those SOAs with large RSEs (cf. Table 1). It is interesting to note that violations of the race model are much larger for B.D. than for K.Y., although even K.Y. shows some violations. Since K.Y. showed only a small redundant signals effect, however, it is not surprising that she also showed only small violations of the race model.

An important statistical question is whether the $V(\mathrm{Ob}-$ served) values in Table 2 might have been observed by chance (sampling error) when in fact a race model was correct. In previous studies, CDFs have usually been obtained for many subjects, and statistical testing was performed by evaluating the consistency of the violations across subjects. Since only 2 subjects were tested in this experiment, however, that procedure would have had unacceptably low power.

To find out how large a $V($ Observed ) might be observed by chance if a race model were actually correct, computer simulation was used (cf. Diaconis \& Efron, 1983). The entire experiment was simulated 1,000 times for each subject, with the following procedure carried out within each simulation. Simulated RTs for the single-signal conditions were obtained by randomly sampling (with replacement) from the observed distributions of single-signal RTs for

Table 2

Violations of Inequality 2 as a Function of SOA and Subject

\begin{tabular}{|c|c|c|c|c|c|c|c|c|c|c|c|}
\hline & \multicolumn{11}{|c|}{ SOA Condition } \\
\hline & \multicolumn{5}{|c|}{$\begin{array}{l}\text { Auditory First: } \\
\text { SOA }_{V}\end{array}$} & \multirow[b]{2}{*}{0} & \multicolumn{5}{|c|}{$\begin{array}{c}\text { Visual First: } \\
\text { SOA A }\end{array}$} \\
\hline & 167 & 133 & 100 & 67 & 33 & & 33 & 67 & 100 & 133 & 167 \\
\hline \multicolumn{12}{|c|}{ Subject B.D. } \\
\hline$V($ Observed) & 0 & 0 & 4 & 0 & 0 & 85 & 340 & 440 & 505 & 246 & 88 \\
\hline$V($ Critical) & 13 & 16 & 17 & 20 & 16 & 22 & 33 & 82 & 87 & 62 & 40 \\
\hline Lower Bound $(V)$ & 0 & $\mathbf{0}$ & 0 & 0 & 0 & 6 & 230 & 353 & 411 & 137 & 22 \\
\hline Upper Bound $(V)$ & 7 & 12 & 36 & 11 & 29 & 132 & 385 & 497 & 552 & 294 & 118 \\
\hline \multicolumn{12}{|c|}{ Subject K.Y. } \\
\hline$V($ Observed $)$ & 0 & 0 & 0 & 6 & 0 & 13 & 46 & 150 & 156 & 10 & 3 \\
\hline$V($ Critical $)$ & 10 & 11 & 12 & 11 & 12 & 17 & 66 & 85 & 69 & 30 & 13 \\
\hline Lower Bound( $V)$ & 0 & 0 & 0 & 0 & 0 & 0 & 4 & 90 & 76 & 0 & 0 \\
\hline Upper Bound $(V)$ & 3 & 6 & 18 & 32 & 4 & 52 & 90 & 218 & 215 & 46 & 41 \\
\hline
\end{tabular}

Note- $V\left(\right.$ Observed) is the area below $F_{R, \text { soA }}(t)$ but above $F_{V}\left(t-\mathrm{SOA}_{V}\right)+F_{A}\left(t-\mathrm{SOA}_{A}\right)$, in violation of Inequality 2 . This area was measured in units of $1 / 1,000$ th of the total area below $F_{R, \text { soA }}(t) . V$ (Critical) is the maximum size of $V($ Observed) that would be expected by chance if race models were correct. Lower and Upper Bound $(V)$ are $95 \%$ confidence limits around $V(\mathrm{Ob}-$ served). For further details see text. 
that subject. In accordance with race models, simulated RTs for the redundant-signal conditions were obtained by sampling two RTs (one from each single-signal distribution), adjusting them appropriately for SOA and selecting the minimum adjusted RT. These two single-signal times were not sampled independently, however. As noted in Inequality 1 , race models predict $F_{R}(t)$ to be at most the sum of $F_{V}(t)$ and $F_{A}(t)$, and this maximum is attained only when the times for racers on the two channels have a strong negative correlation (see Miller, 1982, p. 253; Ulrich \& Giray, 1986, Table 1). Thus, sampling error is most likely to produce violations of Inequality 2 in a race model with a strong negative correlation. To provide a conservative test, making it difficult to reject race models, a strong negative correlation was therefore introduced into the simulation. This was accomplished by randomly selecting an RT from the distribution of visualsignal RTs, computing its percentile, $P$, and then sampling the auditory-signal RT with percentile $100-P$. Thus, relatively fast responses to visual signals were paired with relatively slow responses to auditory signals, and vice versa, producing a strong negative correlation between racers. After sampling the appropriate number of trials for each condition in the simulated experiment, the CDFs for the various conditions and the size of the violation of Inequality 2 were computed from the simulated RTs just as they had been computed from the observed RTs.

To summarize the results of the 1,000 simulated experiments, a critical violation, $V(C r i t i c a l)$, was obtained for each subject and condition. This value was the largest number such that, across the 1,000 simulations, violations larger than that number were obtained only $5 \%$ of the time. This procedure provides a $V$ (Critical) corresponding to the traditional notion of a critical value for any hypothesis-testing statistic, in the sense that an observed violation greater than the critical value will be observed by chance only $5 \%$ of the time if the null hypothesis (i.e., a race model) is correct. Thus, race models can be rejected for any SOA at which $V$ (Observed) is greater than $V(C r i t i c a l)$, with $95 \%$ confidence that the race model is inappropriate for that subject and SOA. The summary values of $V$ (Critical) are shown in Table 2.

On the basis of the results of the computer simulations, it is clear that the observed violations of race models are much larger than would be expected by chance in the conditions with visual signals preceding auditory signalsprecisely those conditions in which RSEs were obtained. Thus, race models can be confidently rejected as an explanation of the RSE with asynchronous as well as simultaneous (Miller, 1982) redundant signals.

Having ruled out race models, it is of interest to consider which SOAs produced the most coactivation (i.e., greatest violation of race models). The $V$ (Observed) values in Table 2 also suggest that the size of the violation varies systematically with $\mathrm{SOA}_{A}$ when the visual signal is presented first. For both subjects, total violation increases with $S A_{A} s$ from 0 to $100 \mathrm{msec}$ and then falls off for
$\mathrm{SOA}_{A} \mathrm{~s}$ of 133 and $167 \mathrm{msec}$. Unfortunately, there is also a statistical problem with the comparison of these observed violations: Since we do not know how far each $V(\mathrm{Ob}-$ served) might be from the true value of the violation for that condition (due to sampling error), we do not know how far apart two values must be before we conclude that the two conditions really have significantly different amounts of violation. To answer this question, a bootstrapping procedure (e.g., Efron, 1979) was used to compute $95 \%$ confidence intervals for the true value of the violation in each condition. Once these confidence intervals were obtained, conditions could be compared by seeing whether their confidence intervals overlapped.

In essence, the bootstrapping procedure takes the observed data as the underlying probability distribution, and determines how much variation in the computed statistic [here, V(Observed)] will be produced by random sampling from that distribution. To carry out the bootstrapping process, the experiment was again simulated 1,000 times for each subject. In these simulations, the RTs for each condition were obtained by randomly sampling (with replacement) from the observed distribution of RTs from that condition. After sampling the appropriate number of RTs from each condition, the CDFs and violations of Inequality 2 for that simulated experiment were computed with the same procedures used to compute these statistics from the observed data. To summarize this set of 1,000 simulations of the experiment, estimates of the upper and lower confidence bounds were chosen. Within a condition, the estimate of the upper bound was the size of violation such that only $2.5 \%$ of the simulated experiments produced violations larger than that. Similarly, the estimate of the lower bound was the size of violation such that only $2.5 \%$ of the simulations produced smaller violations. Together, these two bounds define the middle $95 \%$ of all violations that would be expected if the observed data were, in fact, the true underlying distributions, and it is reasonable to use these values to estimate the $95 \%$ confidence interval for the true $V(O b s e r v e d)$.

The upper and lower confidence bounds obtained by bootstrapping are also shown in Table 2 . Since the confidence intervals around some of the observed violations do not overlap, we can conclude that some of the differences in observed violations are too large to have been obtained by chance, and that some SOAs really produce larger violations of race models than others. It appears that race models are most seriously violated in the conditions with auditory signals presented $67-100 \mathrm{msec}$ after visual signals, for both subjects.

It is interesting to observe that the SOAs producing the largest violations of race models are approximately those SOAs that equalize mean RTs to single signals (corrected for $\mathrm{SOA}$ ), with violations decreasing somewhat symmetrically for SOAs larger and smaller than the one yielding the maximum. This observation is quite consistent with coactivation models. If signals coactivated the response, one would expect maximal coactivation to be found with an SOA for which the two signals produced response ac- 
tivation simultaneously. This suggests that the optimal SOA for violations of race models would be the one that maximizes the overlap of RT distributions to the single signals, since signals producing responses over the same range of times would presumably be producing response activation over the same temporal range as well. If SOA deviates from this optimal value in either direction, there would be less opportunity for coactivation because most of the response activation would come from the signal with the faster mean single signal RT (corrected for SOA).

Before leaving the evaluation of race models, it is also necessary to consider the possibility that the violations observed in Table 2 were caused by artifacts arising from the use of RT distributions obtained by pooling together all the RTs from a given condition. RTs are known to depend on both practice (Fitts, 1964) and sequential effects (Kornblum, 1969), and both of these types of effects were significant in the present experiment. Thus, it is possible that the observed violations somehow resulted from combining different types of responses into a single overall distribution. For example, a response to redundant signals might benefit from stimulus repetition no matter which signal was presented on the previous trial, whereas a response to a single signal could benefit only if preceded by the same signal. Thus, responses to redundant signals would benefit from the repetition effect on a higher proportion of trials than responses to either single signal, and this might explain the failure of race models.

To test for the presence of pooling artifacts, values analogous to those shown in Table 2 were recomputed with more restricted pooling of RTs into overall distributions. Table 3 shows the violations of Inequality 2 obtained when pooling separately across the first and second halves of the experiment for each subject. Table 4 shows the violations obtained when responses were sorted on the basis of whether the previous trial was a catch trial or a redundant signals trial (there were not enough trials following single visual and auditory signals for a meaningful analysis). Clearly, there are significant violations of race models regardless of how the trials are partitioned. Violations were present in both halves of practice and for both types of preceding signals. We can conclude that pooling artifacts of these types do not contribute substantially to the inconsistency with race models.

To summarize the findings from the above analyses, it is clear that race models cannot be used to explain the RSE obtained in this experiment. Comparing Tables 1 and 2 , it is obvious that the predictions of race models were seriously violated at all SOAs for which there was a sizable RSE. Thus, it can be concluded that a response to a redundant signal is not simply a response to either its visual or its auditory component.

\section{Comparison of Accumulation and Exponential Coactivation Models}

Not only do the violations of Inequality 2 contradict race models; they also support coactivation models in which two redundant signals somehow combine to activate the response. Therefore, it is reasonable to compare the accumulation and exponential coactivation models using Inequality 3 .

To evaluate exponential models, Inequality 3 was tested in the same way as Inequality 2, except for the use of a different sum of CDFs on the right-hand side of the inequality. Table 5 shows the total violations of the inequality and the associated critical values and bounds of $95 \%$ confidence intervals, all of which were computed with the same procedures used to obtain the values in Tables 2-4.

The data show substantial violations of the inequality derived from exponential coactivation models in conditions with visual signals presented first. Responses to asynchronous redundant signals were much too fast to have been activated solely by either the visual signal present from time 0 to time $S O A_{A}$ or the redundant signals present after time $\mathrm{SOA}_{A}$. This demonstrates that two signal configurations present at different times can jointly activate the response, so response activation must be accumulated across time as assumed by accumulation models. Apparently, the visual signal gets the response activation process started, and the redundant signals provide the final activation to satisfy the criterion.

In fact, the violations of Inequality 3 are about the same size as violations of Inequality 2 . This suggests that the main source of coactivation-hence, violations of race models-is accumulation of activation over time. One might have hypothesized instead that violations of Inequality 2 were mainly due to an interactive effect of the two signals on the rate at which response activation accumulated. For example, suppose response activation accumulated at the rates of 10,12 , and $5,000,000$ units/sec for visual, auditory, and redundant signals, respectively. This would result in huge violations of Inequality 2, but only very small violations of Inequality 3 . The fact that the violations of Inequality 3 are almost as large as those of Inequality 2 suggests that the rate of response activation for redundant signals is of the same order of magnitude as the sum of the rates for the two single signals.

\section{How Soon Does Response Activation Begin and How Long Does It Take to Reach Criterion?}

Assuming that violations of Inequality 2 reflect coactivation, they can be used to estimate two parameters describing the timecourse of response activation produced by each signal: $O_{s}$, the minimum time from presentation of the signal to the onset of response activation produced by that signal, and $D_{s}$, the minimum accumulation time needed to reach criterion by accumulating response activation just from that signal.

These analyses require comparison of violations across a number of SOAs, so they were only performed with Subject B.D.'s data from conditions with the visual signal presented first. Obviously no general conclusions can be drawn from the results of a single subject tested with specific stimuli, but the comparison of these values in this case leads to some intriguing hypotheses. 
Table 3

Violations of Inequality 2 as a Function of SOA, Practice, and Subject

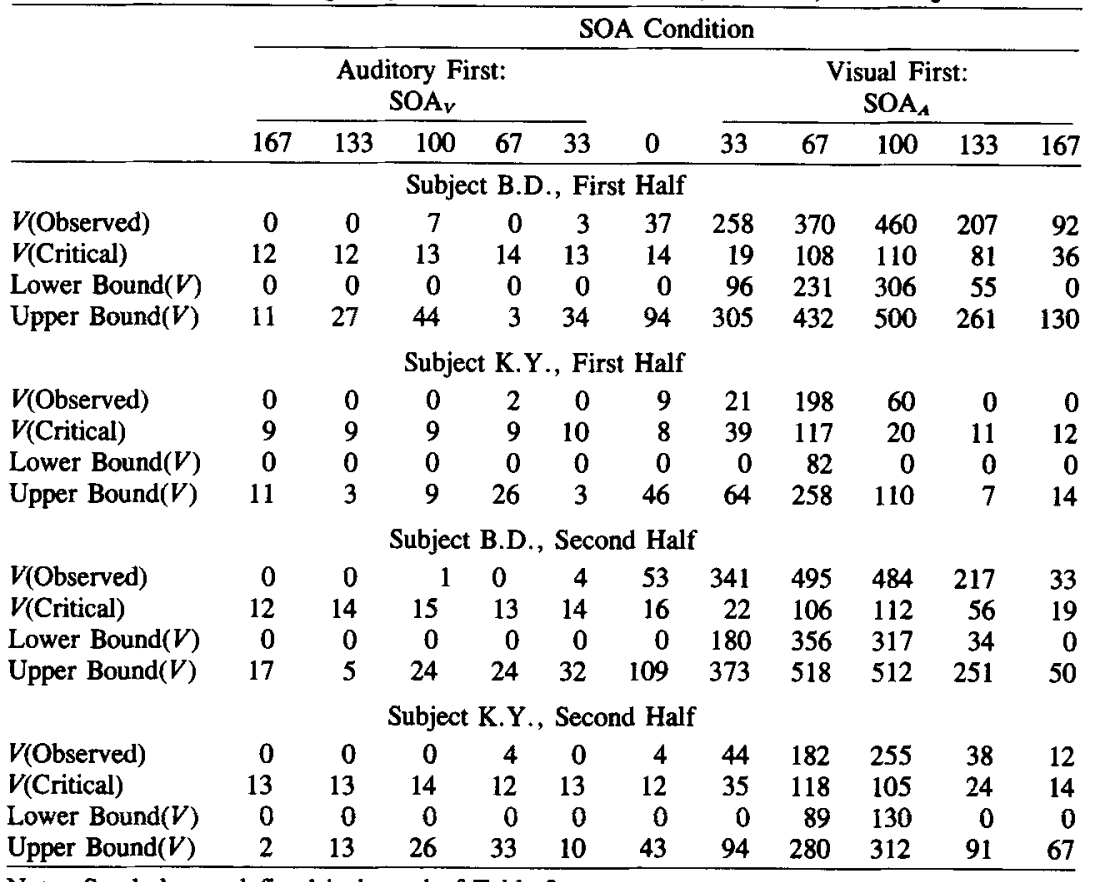

Note-Symbols are defined in legend of Table 2.

Table 4

Violations of Inequality 2 as a Function of SOA, Previous Trial, and Subject

\begin{tabular}{|c|c|c|c|c|c|c|c|c|c|c|c|}
\hline & \multicolumn{11}{|c|}{ SOA Condition } \\
\hline & \multicolumn{5}{|c|}{$\begin{array}{c}\text { Auditory First: } \\
\text { SOA }_{V}\end{array}$} & \multirow[b]{2}{*}{$\underline{0}$} & \multicolumn{5}{|c|}{$\begin{array}{c}\text { Visual First: } \\
\text { SOA }_{A}\end{array}$} \\
\hline & 167 & 133 & 100 & 67 & 33 & & 33 & 67 & 100 & 133 & 167 \\
\hline \multicolumn{12}{|c|}{ Subject B.D., Following Catch Trial } \\
\hline$V($ Observed $)$ & $\mathbf{0}$ & 7 & 16 & 7 & 15 & 67 & 324 & 327 & 270 & 15 & 0 \\
\hline$V($ Critical) & 11 & 12 & 12 & 14 & 13 & 12 & 17 & 76 & 10 & 4 & 4 \\
\hline Lower Bound $(V)$ & 0 & 0 & 0 & $\mathbf{0}$ & 0 & 0 & 7 & 6 & 7 & 0 & 0 \\
\hline Upper Bound $(V)$ & 16 & 30 & 47 & 27 & 49 & 85 & 224 & 228 & 210 & 38 & 7 \\
\hline \multicolumn{12}{|c|}{ Subject K.Y., Following Catch Trial } \\
\hline$V($ Obser & 0 & 0 & 0 & 0 & 2 & 0 & 9 & 79 & 6 & 0 & 0 \\
\hline$V(\mathrm{Cri}$ & 7 & 6 & 6 & 6 & 6 & 1 & 24 & 58 & 6 & $f$ & 7 \\
\hline Lower Bound $(V)$ & 0 & 0 & 0 & 0 & 0 & 0 & $\mathbf{0}$ & 0 & 0 & 0 & 0 \\
\hline Upper Bound $(\boldsymbol{V})$ & 10 & 3 & 4 & 12 & 26 & 13 & 48 & 127 & 33 & 2 & 2 \\
\hline \multicolumn{12}{|c|}{ Subject B.D., Following Redundant Signal Trial } \\
\hline$V(\mathrm{Obs}$ & 0 & 0 & 4 & 0 & 0 & 17 & 237 & 416 & 538 & 253 & 106 \\
\hline$V($ Critical) & 16 & 14 & 11 & 13 & 13 & 15 & 16 & 87 & 106 & 87 & 47 \\
\hline Lower Bound $(V)$ & $\mathbf{0}$ & 0 & 0 & 0 & 0 & 0 & 108 & 292 & 396 & 112 & 9 \\
\hline Upper Bound( $V)$ & 16 & 5 & 33 & 11 & 12 & 67 & 280 & 463 & 560 & 306 & 139 \\
\hline \multicolumn{12}{|c|}{ Subject K.Y., Following Redundant Signal Trial } \\
\hline$V($ Observed $)$ & 0 & 0 & 3 & 22 & 0 & 18 & 48 & 156 & 221 & 11 & 20 \\
\hline$V($ Critical $)$ & 12 & 10 & 10 & 11 & 12 & 11 & 55 & 107 & 81 & 20 & 12 \\
\hline Lower Bound $(V)$ & 0 & 0 & 0 & 0 & 0 & 0 & $\mathbf{0}$ & 77 & 88 & 0 & 0 \\
\hline Upper Bound( $V)$ & 5 & 14 & 37 & 60 & 2 & 63 & 116 & 242 & 272 & 55 & 69 \\
\hline
\end{tabular}

Note-Symbols are defined in legend of Table 2. 
Table 5

Violations of Inequality 3 as a Function of SOA and Subject

\begin{tabular}{|c|c|c|c|c|c|c|c|c|c|c|c|}
\hline & \multicolumn{11}{|c|}{ SOA Condition } \\
\hline & \multicolumn{5}{|c|}{$\begin{array}{l}\text { Auditory First: } \\
\text { SOA }_{V}\end{array}$} & \multirow[b]{2}{*}{0} & \multicolumn{5}{|c|}{$\begin{array}{l}\text { Visual First: } \\
\text { SOA }_{A}\end{array}$} \\
\hline & 167 & 133 & 100 & 67 & 33 & & 33 & 67 & 100 & 133 & 167 \\
\hline \multicolumn{12}{|c|}{ Subject B.D. } \\
\hline$V$ (Observed) & 0 & 0 & 4 & 0 & 0 & & 236 & 374 & 481 & 232 & 85 \\
\hline$V($ Critical) & 16 & 15 & 16 & 15 & 78 & & 26 & 85 & 89 & 67 & 40 \\
\hline Lower Bound $(V)$ & 0 & 0 & 0 & 0 & 0 & & 137 & 293 & 400 & 135 & 29 \\
\hline Upper Bound( $V$ ) & 7 & 10 & 33 & 10 & 14 & & 275 & 438 & 537 & 285 & 116 \\
\hline \multicolumn{12}{|c|}{ Subject K.Y. } \\
\hline$V($ Observed $)$ & 0 & 0 & 0 & 5 & 0 & & 12 & 133 & 129 & 1 & 1 \\
\hline$V($ Critical $)$ & 11 & 12 & 13 & 14 & 51 & & 71 & 96 & 62 & 22 & 10 \\
\hline Lower Bound $(V)$ & 0 & 0 & 0 & 0 & 0 & & 0 & 76 & 44 & 0 & 0 \\
\hline Upper Bound( $V)$ & 4 & 4 & 14 & 27 & 4 & & 57 & 194 & 173 & 23 & 27 \\
\hline
\end{tabular}

Note-V(Observed) is the area below $F_{R, \text { SOA }}(t)$ but above $F_{V}(t)+F_{R, 0}\left(t-S_{\text {SOA }}\right)$ or $F_{A}(t)+$ $F_{R, 0}\left(t-\mathrm{SOA}_{V}\right)$, as appropriate, in violation of Inequality 3 . This area was measured in units of $1 / 1,000$ th of the total area below $F_{R}$, son $(t)$. $V$ (Critical) is the maximum size of $V$ (Observed) that would be expected by chance if exponential models were correct. Lower and Upper Bound $(V)$ are $95 \%$ confidence limits around $V$ (Observed). For further details see text.

$O_{s}$ indicates how soon after its onset each signal begins to produce response activation. To estimate the relative values of $O_{V}$ and $O_{A}$, it is reasonable to use the smallest values of $t$ for which Inequality 2 is violated. If the inequality is violated at time $t$, then both signals must have contributed some activation to responses made at time $t$. Allowing for the necessary motor delay (M), this implies that both signals must have produced some activation by time $t-M$. By finding the smallest value of $t$ producing coactivation, we can find smallest $t-M$ at which activation has been produced by the signal (i.e., the onset of activation).

The middle column of Table 6 shows, for each condition in which the visual signal was presented first, the smallest value of $t$ for which Inequality 2 was violated. In each condition, this was defined as the smallest $t$ for which $F_{R, \operatorname{SOA}}(t)$ was at least .01 greater than the sum of $F_{V}(t)$ and $F_{A}\left(t-S_{S A}\right)$. The smallest value across all conditions was obtained in the conditions with SOAs of 0 and $33 \mathrm{msec}$. In these conditions, the earliest sign of coactivation was in responses with latencies of about 196-197 msec. Thus it appears that a visual signal did not influence a response that occurred less than about $196 \mathrm{msec}$ after it, so it is reasonable to conclude that visual signals did not start to produce any response activation in the first $196-M$ msec. The true value of $O_{v}$ is probably slightly less than $196-M$, however, because the signal might produce a little response activation without causing a violation of Inequality 2 .

The rightmost column of Table 6 shows the analogous values for the auditory signal, measured from the onset of the auditory signal to the point of coactivation (i.e., $t-\mathrm{SOA}_{A}$ ). In the condition with an $\mathrm{SOA}_{A}$ of $33 \mathrm{msec}$, for example, the same responses demonstrating coactivation $197 \mathrm{msec}$ after the visual signal also demonstrated coactivation only 164 msec after the auditory signal, since the latter signal had been delayed by $33 \mathrm{msec}$. This value in- dicates that an auditory signal can influence a response that occurs within $164 \mathrm{msec}$ after it. Values obtained for the other SOAs indicate that an auditory signal can influence a response that occurs as little as $105 \mathrm{msec}$ after it is presented. This implies that the auditory signal must have produced some response activation within the first $105-M$ msec after its onset, since its activation contributed to a violation of race models within that time.

Comparing the relative values of $O_{V}$ and $O_{A}$ just estimated, the conclusion is that an auditory signal can start producing response activation about $91 \mathrm{msec}(196-M$ $-105+M$ ) faster than a visual one. That is, the conclusion is that the minimum time needed for a visual signal to influence a response is much more than that needed for an auditory one. This conclusion is consistent with the faster overall RTs to single auditory signals, and may be one of the reasons why auditory signals are more effective alerting stimuli than visual signals (Posner, Nis-

Table 6

Estimates of Minimum Onset and Duration of Response Activation for B.D.

\begin{tabular}{|c|c|c|}
\hline \multirow[b]{2}{*}{$\begin{array}{c}\text { SOA } \\
\text { (Visual First) }\end{array}$} & \multicolumn{2}{|c|}{ Smallest $t$ Violating Inequality $3^{*}$} \\
\hline & $\begin{array}{l}\text { Measured From } \\
\text { Onset of } \\
\text { Visual Signal }\end{array}$ & $\begin{array}{l}\text { Measured From } \\
\text { Onset of } \\
\text { Auditory Signal }\end{array}$ \\
\hline 0 & 196 & 196 \\
\hline 33 & 197 & 164 \\
\hline 67 & 225 & 158 \\
\hline 100 & 233 & 133 \\
\hline 133 & 238 & 105 \\
\hline 167 & 319 & 152 \\
\hline Estimated Minimum Ons & 196 & 105 \\
\hline Minimum $\mathrm{RT} \dagger$ to Single Signal & 247 & 176 \\
\hline Estimated Minimum Duration & 51 & 71 \\
\hline
\end{tabular}

*Smallest $t$ for which $F_{R, \text { sos }}(t)>F_{A}\left(t-\mathrm{SOA}_{A}\right)+F_{V}(t)+0.01$. †RT at 2.5 percentile was used rather than minimum, to avoid contamination from anticipation responses. 
sen, \& Klein, 1976). It is also interesting to note that this analysis requires $M$ to be smaller than $105 \mathrm{msec}$.

By extending the above argument, it is possible to estimate $D_{V}$ and $D_{A}$, the minimum times needed for each signal to produce enough activation to reach criterion. The minimum duration of response activation can be estimated by the difference between the minimum single-signal RT and the minimum time needed for the onset of activation, estimated above. For example, an auditory signal could influence a response that occurred as little as $105 \mathrm{msec}$ after it, but could never cause a response all by itself in less than $176 \mathrm{msec}$ (minimum RT for trials with a single auditory signal). From this comparison, it appears that auditory signals required at least $71 \mathrm{msec}(176-105)$ to produce enough activation to reach criterion.

Comparable values for the visual signal were, surprisingly, somewhat shorter. The fastest responses to a visual signal $(247 \mathrm{msec})$ were only $51 \mathrm{msec}$ slower than the fastest responses that could be influenced by a visual signal $(196 \mathrm{msec})$. Thus, it appears that the visual signal can produce enough activation to reach criterion in less time (measured from the onset of activation) than the auditory signal, even though the onset of activation is earlier for the auditory signal. It should be emphasized that these comparisons are between minimum times, and the same conclusions may not apply to average time needed for activation to begin and to reach criterion. However, if the assumptions of the analysis are correct, the values of 71 and $51 \mathrm{msec}$ are estimates of the minimum absolute duration of response activation, not just relative values.

\section{The Combination Rule of Grice, Canham, and Boroughs (1984)}

Grice et al. (1984) have proposed a form of accumulation model that incorporates the qualitative features implied by the above analyses of RT distributions. Their model gave a very good account of results obtained in letter-detection tasks, accounting for over $99 \%$ of the variance. Thus, it seemed reasonable to fit the model to the present data. The model is described briefly below, and then the results of the fit to the present data are reported.

In Grice et al.'s (1984) model, response activation $E(t)$ grows deterministically as a function of time since onset of the signal, without random trial-to-trial variation. When response activation reaches a criterion, the response is initiated. Variation in RT is caused by the fluctuation of this response criterion, which is assumed to have a normal distribution with unknown mean and variance.

In a divided-attention task, it is natural to assume that signals on both channels produce response activation, and that the total activation produced by redundant signals is some function of the activations produced by the two single signals. Grice et al. (1984) used the observed cumulative density functions of RT to estimate $E_{1}(t), E_{2}(t)$, and $E_{R}(t)$, the functions describing the timecourse of the response activation produced by a signal on channel 1 , a signal on channel 2 , and redundant signals, respectively (see Grice et al., 1984, for a fuller description). They then used linear regression to look for a combination rule that would predict $E_{R}(t)$ from $E_{1}(t)$ and $E_{2}(t)$. In Grice et al.'s (1984) data, a linear averaging function accounted for about $99 \%$ of the variance in each of several experiments:

$$
E_{R}(t)=C+A \times\left[E_{1}(t)+E_{2}(t)\right] .
$$

In several experiments, the values of $\boldsymbol{A}$ were consistently about 0.5 , and $C$ ranged from about .3 to .6 .

Grice et al.'s (1984) model was fit separately for each subject and redundant-signal condition of the present experiment. First, $E_{R, \mathrm{SOA}}(t)$ was estimated for each of the 11 redundant-signal conditions, using Grice et al.'s Equation 4. Twenty values of $t$ were identified in each condition, corresponding to $F(t)$ values ranging from 0.025 to 0.975 in steps of 0.05 . Following Grice et al., 3.5 was added to each $E(t)$ to adjust the origins of these activation functions to 0 . Next, using the distributions of RT obtained in each of the two single-signal conditions, $E_{A}\left(\mathrm{t}-\mathrm{SOA}_{A}\right)$ and $E_{V}\left(t-\mathrm{SOA}_{V}\right)$ were computed for each of the 20 values of $t$ from each of the 11 redundant-signal conditions. Then linear regression was used to estimate, for each condition, the values of $A$ and $C$ that best predict the 20 values of $E_{R, \mathrm{sOA}}(t)$ from the sum of the corresponding $E_{A}\left(t-\mathrm{SOA}_{A}\right)$ and $E_{V}\left(t-\mathrm{SOA}_{V}\right)$.

Table 7 summarizes the fits for both subjects across all conditions. These fits are reasonably good for Subject B.D., accounting for a total of $96.6 \%$ of the variance across all conditions, although these fits are not nearly as precise as those obtained by Grice et al. (1984). Inspection of the 11 fits indicated that $E_{R}(t)$ is consistently overpredicted for values of $t$ corresponding to cumulative percentages of $2.5,7.5,12.5$, and 97.5 , and consistently underpredicted at values of $t$ corresponding to percentages from 42.5 to 57.5 . Across the 11 regression analyses, the prediction errors at all of these values of $t$ were consistently different from zero by Student's $t$ tests $(d f=10, p<.05)$.

The fits are much poorer for K.Y., accounting for only $81.7 \%$ of the total variance across conditions. For this subject, too, the model significantly overpredicted $E_{R}(t)$ at the lower values of $t$, corresponding to cumulative percentages of 2.5 to 22.5 , and underpredicted for values of $t$ from 37.5 to 87.5. Inspection of the percent variance accounted for by the model in each condition (Table 7) shows that the model fits especially poorly for K.Y. in the conditions in which no redundant signals effect was found (cf. Table 1).

A more stringent test of the model is to see how well $E_{R, \mathrm{SOA}}(t)$ can be predicted from $E_{A}(t-\mathrm{SOA})+$ $E_{v}\left(T-\mathrm{SOA}_{V}\right)$ using a single set of estimates for $A$ and $C$ across all SOA conditions. Since the different conditions were mixed together within blocks of trials, it is hard to see how the parameter estimates could vary across these conditions. When all 220 points for B.D. were pooled into a single regression equation, the estimates of $C$ and $A$ were 0.83 and 0.66 , respectively, and the model accounted for only $89.7 \%$ of the variance. For K.Y., the estimates were 1.89 and 0.38 , with $76.9 \%$ of the variance accounted for. The reduction in prediction accuracy, compared with the set of models using different values of $C$ and $A$ for the 
Table 7

Estimates of $A$ and $C$ and Percentage of Variance Accounted for as a Function of SOA and Subject

\begin{tabular}{|c|c|c|c|c|c|c|c|c|c|c|c|}
\hline & \multicolumn{11}{|c|}{ SOA Condition } \\
\hline & \multicolumn{5}{|c|}{$\begin{array}{l}\text { Auditory First: } \\
\text { SOA }_{v}\end{array}$} & \multicolumn{6}{|c|}{$\begin{array}{l}\text { Visual First: } \\
\text { SOA }_{A}\end{array}$} \\
\hline & 167 & 133 & 100 & 67 & 33 & 0 & 33 & 67 & 100 & 133 & 167 \\
\hline \multicolumn{12}{|c|}{ Subject B.D. } \\
\hline $\begin{array}{c}\mathrm{C} \\
\mathrm{A} \\
\% \mathrm{Var}\end{array}$ & $\begin{array}{r}.65 \\
.74 \\
97.7\end{array}$ & $\begin{array}{r}-.09 \\
.86 \\
98.1\end{array}$ & $\begin{array}{r}-.55 \\
.97 \\
99.6\end{array}$ & $\begin{array}{r}-.30 \\
.88 \\
99.0\end{array}$ & $\begin{array}{r}-.45 \\
.94 \\
99.3\end{array}$ & $\begin{array}{r}.06 \\
.80 \\
94.8\end{array}$ & $\begin{array}{r}.90 \\
.65 \\
94.8\end{array}$ & $\begin{array}{r}1.02 \\
.59 \\
99.2\end{array}$ & $\begin{array}{r}1.61 \\
.53 \\
95.1\end{array}$ & $\begin{array}{l}1.46 \\
.55 \\
93.5\end{array}$ & $\begin{array}{r}.96 \\
.67 \\
91.4\end{array}$ \\
\hline
\end{tabular}

\begin{tabular}{crrrrrrrrrrr}
\multicolumn{10}{c}{ Subject K.Y. } \\
C & 1.82 & 1.93 & 1.91 & 2.07 & 1.95 & 1.90 & 1.30 & 1.36 & 1.66 & 1.75 & 1.90 \\
A & .45 & .40 & .43 & .36 & .37 & .37 & .41 & .41 & .42 & .40 & .41 \\
\%Var & 67.1 & 69.5 & 65.1 & 73.9 & 77.4 & 86.9 & 97.9 & 97.7 & 94.8 & 89.6 & 77.9 \\
\multicolumn{1}{c}{ Total variance accounted for across all 11 } & conditions $=81.7 \%$ \\
\hline
\end{tabular}

different SOA conditions, was significant for both subjects [B.D., $F(20,198)=20, p<.01, M S_{\mathrm{e}}=0.0354$; K.Y., $\left.F(20,198)=2.57, p<.01, M S_{\mathrm{e}}=0.4845\right]$.

\section{CONCLUSIONS}

The results of this experiment provide further evidence against race models of bimodal detection tasks, extending previous work with simultaneous redundant signals (Miller, 1982). RT distributions obtained from two highly practiced subjects indicate that their responses to sequentially presented redundant signals were too fast to be explained in terms of the winner of a race between two processes responding separately to the two single signals. Violations of race models tended to be larger in conditions in which the RSE was larger, so it is clear that race models cannot account for that effect.

Tests of Inequality 3 showed that, for both subjects, responses to sequentially presented redundant signals were also too fast to have been produced by a race between one process responding to the single signal and a separate process, starting SOA msec later, responding to the redundant signals. Activation appears to have been accumulated over time as well as over signals, so an individual response can sometimes be activated both by the single signal present at the beginning of the trial and by the redundant signals present after the end of the SOA. This finding supports accumulation models of coactivation and of RT processes in general, and it is evidence against exponential models in which the response is generated by history-free processes.

It is perhaps surprising that response processes accumulate activation over a significant period of time, even in simple signal detection tasks with superthreshold signals. The requirement of a long accumulation interval may be a necessary corollary of the need to maintain a fairly high response criterion to avoid making false alarms on catch trials (cf. Grice, 1972).
The results of the present paper, although strongly supporting accumulation models of coactivation, unfortunately give no indication of the level of processing that is responsible for coactivation, and it will be important to try to identify this level in future research. For example, one possible coactivation model would localize the interaction within a central decision process that accumulates evidence of signals until it has enough to satisfy an internal response criterion (Miller, 1982). If each signal causes evidence to accumulate in this decision maker, coactivation could arise because the decision process would pool evidence coming from different signals, allowing both to contribute toward satisfying the response criterion. Naturally, this would produce faster responses on redundant-signal trials than on single-signal trials. Miller (1982) argued for decision-level coactivation on several grounds.

Other possible coactivation models might localize the interaction between signals within the sensory or motor systems, and these models are closely related to the energy summation and preparation enhancement models of the intersensory facilitation observed in focused-attention tasks (e.g., Nickerson, 1973). Energy summation models are based on the idea that energy from different signals sums within the perceptual system, as might be the case if neurons responding to signals on different modalities converged on a common sensory area (cf. Jung, Kornhuber, \& DaFonseca, 1963). Such convergence would produce a stronger perceptual signal on redundant-signals trials than on single-signal trials. Preparation-enhancement models assume that redundant signals combine in activating the motor system, so that motor responding is faster to redundant than to single signals.

\section{REFERENCES}

AshBy, F. G. (1982). Testing the assumptions of exponential, additive reaction time models. Memory \& Cognition, 10, 125-134.

AsHBY, F. G., \& TownSEND, J. (1980). Decomposing the reaction time 
distribution: Pure insertion and selective influence revisited. Journal of Mathematical Psychology, 21, 93-123.

AUDLEY, R. J. (1973). Some observations on theories of choice reaction time: Tutorial review. In S. Kornblum (Ed.), Attention and performance (Vol. 4; pp. 509-545). New York: Academic Press.

BernsteIN, I. H. (1970). Can we see and hear at the same time? Some recent studies of intersensory facilitation of reaction time. Acta Psychologica, 33, 21-35.

Blake, R. R., Martens, W., Garrett, A., \& Westendorf, D. (1980). Estimating probability summation for binocular reaction time data. Perception \& Psychophysics, 27, 375-378.

Christie, L., Luce, R. D. (1956). Decision structure and time relations in simple choice behavior. Bulletin of Mathematical Biophysics, $18,89-112$.

Colavita, F. (1974). Human sensory dominance. Perception \& Psychophysics, 16, 409-412.

Corcoran, D. W. J., \& Wening, D. (1969). On the combination of evidence from the eye and ear. Ergonomics, 12, 383-394.

DiACONIS, P., \& EFRoN, B. (1983). Computer-intensive methods in statistics. Scientific American, 248, 116-130.

EFRON, B. (1979). Bootstrap methods: Another look at the jackknife. Annals of Statistics, 7, 1-26.

FITTs, P. (1964). Perceptual-motor skill learning. In A. Melton (Ed.), Categories of human learning (pp. 243-285). New York: Academic Press.

Gielen, S. C. A. M., Schmid, R. A., \& Van den Heuvel, P. J. M. (1983). On the nature of intersensory facilitation of reaction time. Perception \& Psychophysics, 34, 161-168.

GreEN, D. M., \& SMTTH, A. F. (1982). Detection of auditory signals occurring at random times: Intensity and duration. Perception \& Psychophysics, 31, 117-127.

GrICE, G. R. (1972). Application of a variable criterion model to auditory reaction time as a function of the type of catch trial. Perception \& Psychophysics, 12, 103-107.

Grice, G. R., Canham, L., \& Boroughs, J. M. (1984). Combination rule for redundant information in reaction time tasks with divided attention. Perception \& Psychophysics, 35, 451-463.

HoCKLEY, W. E. (1984). Analysis of response time distributions in the study of cognitive processes. Journal of Experimental Psychology: Leaming, Memory, \& Cognition, 10, 598-615.

HонLе, R. (1965). Inferred components of reaction times as functions of foreperiod duration. Journal of Experimental Psychology, 69, 382-386.

Jung, R., Kornhuber, H., \& DAFonseCA, J. (1963). Multisensory convergence on cortical neurons: Neuronal effects of visual, acoustic and vestibular stimuli in the superior convolutions of the cat's cortex. In G. Moruzzi, A. Fessard, \& H. Jasper (Eds.), Progress in brain research, Vol. 1: Brain mechanisms. Amsterdam: Elsevier.

Kornblum, S. (1969). Sequential determinants of information processing in serial and discrete choice reaction time. Psychological Review, 76, 113-131.

LiNK, S. W. (1975). The relative judgment theory of two choice response time. Journal of Mathematical Psychology, 12, 114-135.

LONG, J. B. (1976). Effect of task difficulty on the division of attention between nonverbal signals: Independence or interaction? Quarterly Journal of Experimental Psychology, 28, 179-192.

LuCE, R. D., \& GREFN, D. M. (1970). Detection of auditory signals presented at random times, I. Perception \& Psychophysics, 7, 1-14.

MCGILL, W. (1963). Stochastic latency mechanisms. In R. D. Luce, R. Bush, \& E. Galanter (Eds.), Handbook of mathematical psychology (Vol. 1, pp. 309-360). New York: Wiley.

Mejers, L., \& Eujkman, E. (1977). Distributions of simple RT with single and double stimuli. Perception \& Psychophysics, 22, 41-48.

MILLER, J. O. (1982). Divided attention: Evidence for coactivation with redundant signals. Cognitive Psychology, 14, 247-279.

Muluigan, R. M., \&Haw, M. L. (1980). Multimodal signal detection: Independent decisions vs. integration. Perception \& Psychophysics, 28, 471-478.

NiCKERSON, R. S. (1973). Intersensory facilitation of reaction time: Energy summation or preparation enhancement. Psychological Review, 80, 489-509.
Posner, M. I., Nissen, M. J., \& Klein, R. (1976). Visual dominance: An information-processing account of its origins and significance. Psychological Review, 83, 157-171.

RAAB, D. (1962). Statistical facilitation of simple reaction times. Transactions of the New York Academy of Sciences, 24, 574-590.

Ratclif, R. (1978). A theory of memory retrieval. Psychological Review, 85, 59-108.

RATCLIFF, R., \& MURDOCK, B. (1976). Retrieval processes in recognition memory. Psychological Review, 83, 190-214.

SHAw, M. L. (1982). Attending to multiple sources of information: I. The integration of information in decision making. Cognitive Psychology, 14, 353-409.

Townsend, J. T., AsHBY, F. G. (1983). The stochastic modeling of elementary psychological processes. New York: Cambridge University Press.

UlRiCh, R., \& GIRAY, M. (1986). Separate-activation models with variable base times: Testability and checking of cross-channel dependency. Perception \& Psychophysics, 39, 248-259.

VICKERS, D. (1970). Evidence for an accumulator model of psychophysical discrimination. Ergonomics, 13, 37-58.

\section{NOTES}

1. Other investigators (e.g., Blake, Martens, Garrett, \& Westendorf, 1980; Meijers \& Eijkman, 1977) have tested race models by making a particular assumption (usually independence) about the correlation between processes responding to signals on different modalities. From such an assumption, one can derive exact predictions for $F_{R}(t)$, thus obtaining tests with more statistical power. Unfortunately, making such an assumption leads to a test with less conceptual power, since, if the predictions are not fulfilled, one does not know whether the race model or the assumed correlation is wrong. The test based on Inequality 1 is preferred here for its generality: If this inequality is violated, no race model is consistent with the data, regardless of the assumed correlation.

2. For the purposes of this paper, many distinguishable RT models are included without distinction in the accumulation class. These are all models in which the decision is made by a process changing states gradually over time, including diffusion models, random walk models, and so forth. The class of accumulation models discussed here should not be confused with the accumulator model of Vickers (1970), which is one particular member of this class.

3. A variant of an accumulation model could assume that the decision process accumulates activation over time, but restarts if the signal conditions change. This would allow accumulation over time, but not combination of activation from an early single signal and a later redundant one. For the purposes of this paper, such a model will be considered to be exponential, because it is severely limited in its ability to accumulate information over time. In any case, this model was ruled out by the experimental results.

Likewise, it is possible for an exponential model to allow an effect of history by assuming that the exponential response rate to a redundant signal depends on whether a single signal was previously present. This assumption seems contrary to the spirit of exponential models, however, since it allows history to influence the decision. Therefore, models of this type will be classed with accumulation models, even if the decision process is exponential, given a static signal configuration.

4. The term "signals" is used here to refer to physical stimuli, not to internal representations that activate responses. An anonymous reviewer rightly pointed out that, if the internal representation of an auditory signal were formed much more quickly than the internal representation of a visual signal, the auditory representation might fully activate the response even though the visual stimulus had been presented first. Since the auditory representation would have arisen as a result of the redundant physical stimulus, though, this consideration does not complicate the interpretation of the inequality derived below.

(Manuscript received May 2, 1986; revision accepted for publication August 25, 1986.) 